University of Nebraska - Lincoln

DigitalCommons@University of Nebraska - Lincoln

\title{
Angular and Energy Dependence of Cross Sections for Ejection of Electrons from Water Vapor. II. 15-150-keV Proton Impact
}

\author{
M. A. Bolorizadeh \\ University of Nebraska - Lincoln \\ M. Eugene Rudd \\ University of Nebraska - Lincoln, erudd@unl.edu
}

Follow this and additional works at: https://digitalcommons.unl.edu/physicsrudd

Part of the Physics Commons

Bolorizadeh, M. A. and Rudd, M. Eugene, "Angular and Energy Dependence of Cross Sections for Ejection of Electrons from Water Vapor. II. 15-150-keV Proton Impact" (1986). M. Eugene Rudd Publications. 9. https://digitalcommons.unl.edu/physicsrudd/9 


\title{
Angular and energy dependence of cross sections for ejection of electrons from water vapor. II. 15-150-keV proton impact
}

\author{
M. A. Bolorizadeh* and M. E. Rudd \\ University of Nebraska-Lincoln, Lincoln, Nebraska 68588-0111
}

(Received 20 May 1985)

\begin{abstract}
Absolute values of cross sections for electron ejection by protons of $15-150 \mathrm{keV}$ energy have been measured as a function of the angle and energy of the electrons. The range of angles was $10^{\circ}$ to $160^{\circ}$ and the electron energy range was $1-300 \mathrm{eV}$. The doubly differential cross sections were also integrated over energy or angle to obtain singly differential and total cross sections and also average ejected electron energies. Good agreement is obtained with Senger's DDCS-MT (doubly differential cross section-mixed treatment) theoretical treatment using the Salin factor.
\end{abstract}

\section{INTRODUCTION}

Measurements of the angular and energy dependence of the double differential cross sections (DDCS) for ejection of electrons in ion-atom collisions have uncovered new mechanisms of ionization ${ }^{1}$ and the availability of such measurements has stimulated theoretical work on ionization. ${ }^{2}$ Unfortunately, while there are partially successful theoretical treatments at high energies (above say, 100 $\mathrm{keV}$ ), the low-energy region remains without an accurate method of calculating electron-ejection cross sections. Thus, there is still a need for measurements in the energy range below the maximum in the total cross-section curve. In searching for systematics in the process, it is desirable to have measurements from a wide variety of targets with various degrees of complexity, different ionization potentials, different outer shell angular momenta, etc.

While low-energy measurements of this kind are available for a number of simple gases, ${ }^{3,4}$ there have been none for molecules more complex than oxygen. Besides its theoretical interest as a triatomic molecule, water vapor is of great practical interest to those modeling radiation damage in living tissue. Toburen and Wilson ${ }^{5}$ have made DDCS measurements on water vapor from 300-1500 $\mathrm{keV}$. The present work extends this range downward into the region where present theory has little to say.

\section{EXPERIMENTAL PROCEDURE}

The apparatus will be only briefly described here since a fuller description is already available elsewhere. ${ }^{1,6}$ Protons accelerated from an if ion source were magnetically analyzed and finely collimated before entering the target gas. A biased Faraday cup caught the proton beam and the collected charge was integrated. Electrons ejected from the static gas (typically at $0.5 \mathrm{mTorr}$ ) entered a $127^{\circ}$ electrostatic analyzer which could be placed at any of the eight angles from $10^{\circ}$ to $160^{\circ}$ relative to the beam direction. The angular acceptance was $2.16^{\circ}$ and the energy resolution $4.4 \%$. No preacceleration of electrons was used. A 17-stage electron multiplier with its first stage held at $80 \mathrm{~V}$ detected individual electrons. The earth's magnetic field was annulled to within a few $\mathrm{mG}$ by the use of three mutually perpendicular pairs of Helmholtz coils.

The efficiency of the detector was determined in an auxiliary measurement using the apparatus of Cacak and Jorgensen ${ }^{7}$ between an electron gun and the electron multiplier detector. Two different apertures, the sizes of which had been carefully measured, could be moved in front of the first dynode. A defocused beam from an electron gun was then directed to the apertures. The uniformity of the beam was checked by moving the smaller aperture across the larger one. The efficiency of the multiplier was determined by comparing the current through the larger aperture, using the first dynode as a Faraday cup, and the count rate through the smaller one when the multiplier was operated in its normal way. By this method the efficiency was found to be $0.63 \pm 0.05$.

Corrections were made for neutralization of the proton beam as it traversed the gas and absorption of the ejected electrons by the target gas, as well as for electrons originating from the background gas. Target pressure was measured with a capacitance manometer. As in earlier work $^{1}$ cross sections differential in angle $\sigma(\theta)$ or energy $\sigma(W)$ were obtained by numerical integration of the DDCS $\sigma(W, \theta)$. Total electron-ejection cross sections $\sigma_{-}$ were calculated by integrating over both energy and angle.

There was a $7 \%$ uncertainty in the measurement of target gas density and an $8 \%$ uncertainty in the detector efficiency. The uncertainties in other quantities were small so the combined uncertainty in the DDCS was $11 \%$ except at the upper end of the electron energy range where low count rates resulted in larger statistical errors. Measurements of the cross sections below about $10 \mathrm{eV}$ may also have additional systematic errors due to deflection of electrons by stray electric and magnetic fields and by the possible presence of spurious low-energy secondary electrons from surfaces. Since the former effect tends to reduce the measured cross sections and the latter to increase them, the results may be either too low or too high. The magnitude of the error is difficult to determine but is probably small above $10 \mathrm{eV}$. However, since low-energy cross sections contribute strongly to $\sigma(\theta)$ and $\sigma_{-}$, there is 
an additional uncertainty in the integrated values which we estimate at $15 \%$ yielding a total uncertainty in $\sigma(\theta)$ and $\sigma_{-}$of about $19 \%$.

\section{EXPERIMENTAL RESULTS}

DDCS are shown in Figs. 1-3. In Fig. 1, showing the electron-energy distributions at various angles, the $10^{\circ}$ curve shows the beginning of the broad binary-encounter peak which is expected to be centered at $327 \mathrm{eV}$. A more prominent peak at about $80 \mathrm{eV}$ can be attributed to electron transfer to the continuum. ${ }^{8,9}$ Interestingly, a small shoulder at the same energy also shows up in the $160^{\circ}$ curve. This peak is similar to the electron-loss peak seen when using projectiles carrying electrons. It is possible that some protons in the beam in this experiment were neutralized in the target gas before reaching the collision center, thus leading to the possibility that this mechanism could explain the $160^{\circ}$ peak. However, when we attempted to verify this by reducing the target-gas pressure, we found no change in the shape of the curve. Since the pressure in the $2-\mathrm{m}$-long beam line was only $1-2 \times 10^{-6}$ Torr, the neutralization of the beam there should not be greater than $1-2 \%$ at low energies and would be far too small at $150 \mathrm{keV}$ to produce a noticeable effect. Another possible explanation is that it is due to a second-order electron transfer to the continuum.

A comparison of the angular distributions of electrons given in Figs. 2 and 3 shows that at $100 \mathrm{keV}$ the electrons are more strongly peaked in the forward direction than at lower impact energies. Also one notes that at the lower impact energy there is a rise in the cross section in the backward direction while there is little if any at $100 \mathrm{keV}$.

Senger ${ }^{10,11}$ has made calculations of the singly and doubly differential cross sections for electron ejection from

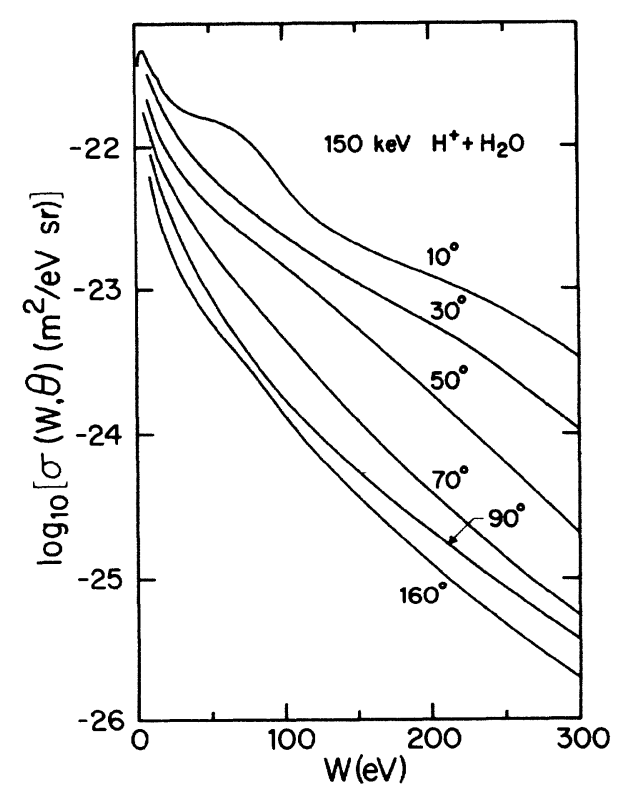

FIG. 1. Doubly differential cross sections for ejection of electrons by $150-\mathrm{keV}$ protons from water vapor as a function of ejection energy for various angles of ejection.

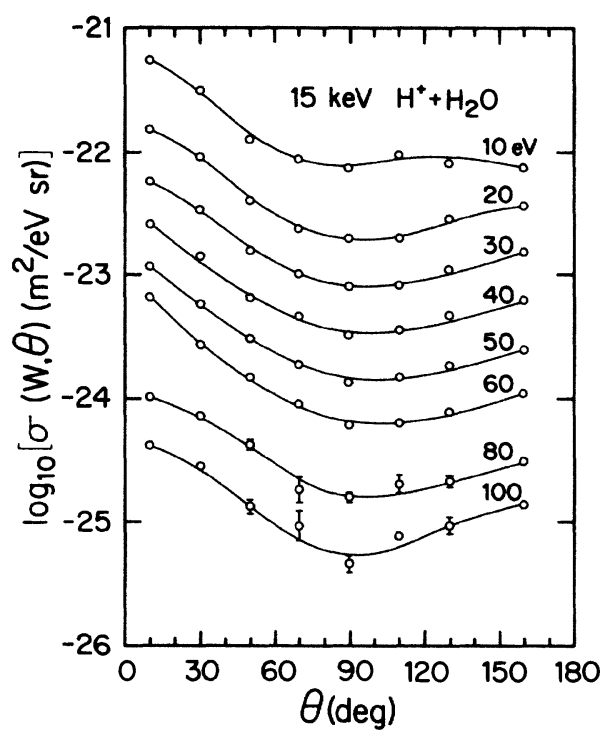

FIG. 2. Doubly differential cross sections for ejection of electrons by $15-\mathrm{keV}$ protons from water vapor as a function of angle of ejection for various ejection energies. Error bars on some data points show the error due to statistical fluctuations in the count.

proton impact on water molecules. He applied the planewave Born approximation to the various molecular orbitals, modifying the form factors and making corrections for binding energies of inner shells. In addition, he has applied the Salin factor ${ }^{12}$ to account for the mechanism of electron transfer to the continuum. His results are com-

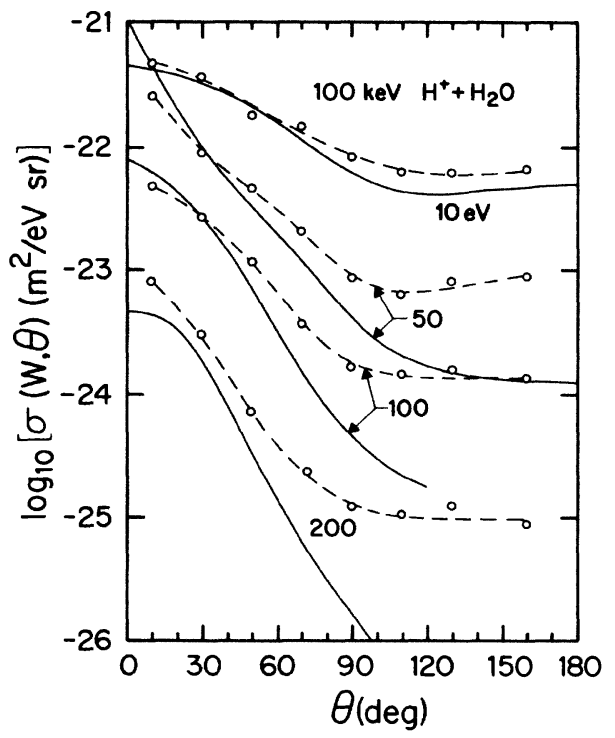

FIG. 3. Doubly differential cross sections for ejection of electrons by $100-\mathrm{keV}$ protons from water vapor as a function of angle. Points are experimental values with the dashed lines drawn to guide the eye. Solid lines are theoretical calculations by Senger et al. (Refs. 10 and 11). 
TABLE I. Values of $\sigma(W)$ in $\mathrm{m}^{2} / \mathrm{eV}, \sigma_{-}$in $\mathrm{m}^{2}$, and $W_{\mathrm{av}}$ in $\mathrm{eV}$ for $\mathrm{H}^{+}+\mathrm{H}_{2} \mathrm{O}$ collisions.

\begin{tabular}{|c|c|c|c|c|c|c|c|}
\hline \multirow[b]{2}{*}{$W(\mathrm{eV})$} & \multicolumn{7}{|c|}{ Projectile energy (keV) } \\
\hline & 15 & 20 & 30 & 50 & 70 & 100 & 150 \\
\hline 2 & $1.5(-21)^{\mathrm{a}}$ & $2.1(-21)$ & $2.2(-21)$ & $2.2(-21)$ & $2.6(-21)$ & $1.6(-21)$ & $1.6(-21)$ \\
\hline 3 & $2.1(-21)$ & $2.4(-21)$ & $2.5(-21)$ & $2.3(-21)$ & $2.5(-21)$ & $2.2(-21)$ & $1.8(-21)$ \\
\hline 5 & $2.5(-21)$ & $2.8(-21)$ & $2.7(-21)$ & $2.5(-21)$ & $2.5(-21)$ & $2.3(-21)$ & $2.2(-21)$ \\
\hline 8 & $2.0(-21)$ & $2.2(-21)$ & $2.4(-21)$ & $2.2(-21)$ & $2.1(-21)$ & $1.9(-21)$ & $1.7(-21)$ \\
\hline 13 & $1.0(-21)$ & $1.2(-21)$ & $1.7(-21)$ & $1.6(-21)$ & $1.5(-21)$ & $1.4(-21)$ & $1.2(-21)$ \\
\hline 20 & $4.5(-22)$ & $6.2(-22)$ & $8.8(-22)$ & $1.2(-21)$ & $1.1(-21)$ & $9.4(-22)$ & $7.7(-22)$ \\
\hline 30 & $1.8(-22)$ & $2.8(-22)$ & $4.4(-22)$ & $6.7(-22)$ & $7.4(-22)$ & $6.4(-22)$ & $5.0(-22)$ \\
\hline 50 & $3.2(-23)$ & $6.2(-23)$ & $1.3(-22)$ & $2.5(-22)$ & $3.2(-22)$ & $3.6(-22)$ & $2.8(-22)$ \\
\hline 80 & $3.8(-24)$ & $8.2(-24)$ & $2.5(-23)$ & $7.4(-23)$ & $1.1(-22)$ & $1.4(-22)$ & $1.4(-22)$ \\
\hline 130 & $6.7(-25)$ & $8.1(-25)$ & $2.0(-24)$ & $9.5(-24)$ & $2.3(-23)$ & $4.3(-23)$ & $4.8(-23)$ \\
\hline 200 & $1.9(-25)$ & $2.0(-25)$ & $3.1(-25)$ & $7.9(-25)$ & $2.6(-24)$ & $8.7(-24)$ & $1.6(-23)$ \\
\hline 300 & $5.6(-26)$ & $7.2(-26)$ & $9.3(-26)$ & $1.1(-25)$ & $2.2(-25)$ & $7.1(-25)$ & $3.0(-24)$ \\
\hline$\sigma_{-}$ & $3.2(-20)$ & $4.0(-20)$ & $5.1(-20)$ & $6.0(-20)$ & $6.4(-20)$ & $6.2(-20)$ & $5.5(-20)$ \\
\hline $\boldsymbol{W}_{\mathrm{av}}$ & 12.33 & 13.6 & 16.7 & 22.4 & 26.0 & 32.2 & 37.0 \\
\hline
\end{tabular}

a The designation $1.4(-22)$ means $1.4 \times 10^{-22}$.

pared with the present experimental data in Fig. 3. While the Salin factor improves the agreement at the forward angles, it worsens the agreement at angles greater than $90^{\circ}$.

SDCS are shown in Figs. 4-6 and in Table I. While there are no earlier experiments in our energy range with which we can compare directly, plots may be made of the SDCS versus proton energy to compare with the corresponding data of Toburen and Wilson ${ }^{5}$ as shown in Fig. 4. While the trend of their data at the lowest energies indicates an extrapolation to somewhat lower values than our data, the general agreement is satisfactory.

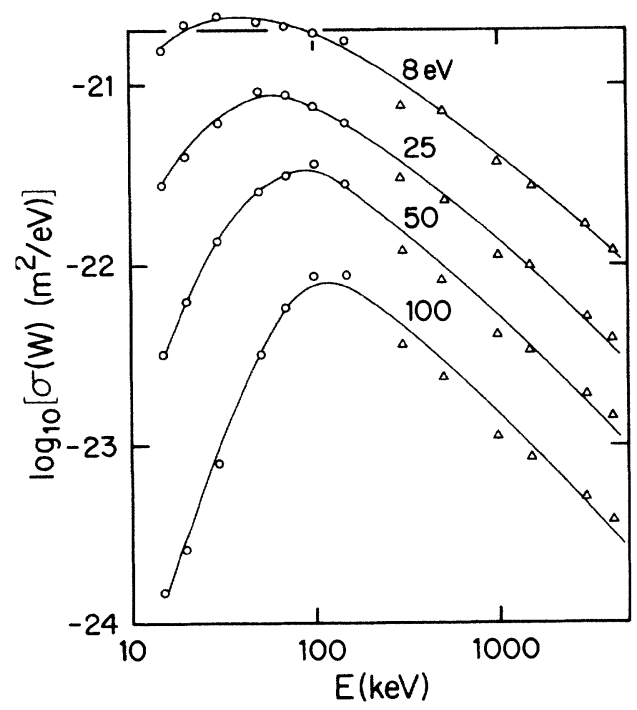

FIG. 4. Singly differential cross sections for ejection of electrons by protons in water vapor as a function of proton energy. Circles, present data; triangles, data of Toburen and Wilson (Ref. 5).
Senger's calculations of SDCS are shown in Fig. 5 where they are compared to the experimental values. Also plotted are calculations made using the binary encounter model ${ }^{13}$ integrated over a Fock distribution of orbital energies. ${ }^{14}$ Partial cross sections for each molecular subshell were added to obtain the cross sections shown. Senger's calculations are in excellent agreement with experiment in this case while the binary-encounter approximation (BEA) overestimates the cross sections in the low-energy region.

Figure 6 shows a comparison of the SDCS versus electron energy for various impact energies. While in every case we took the precaution of taking a background count

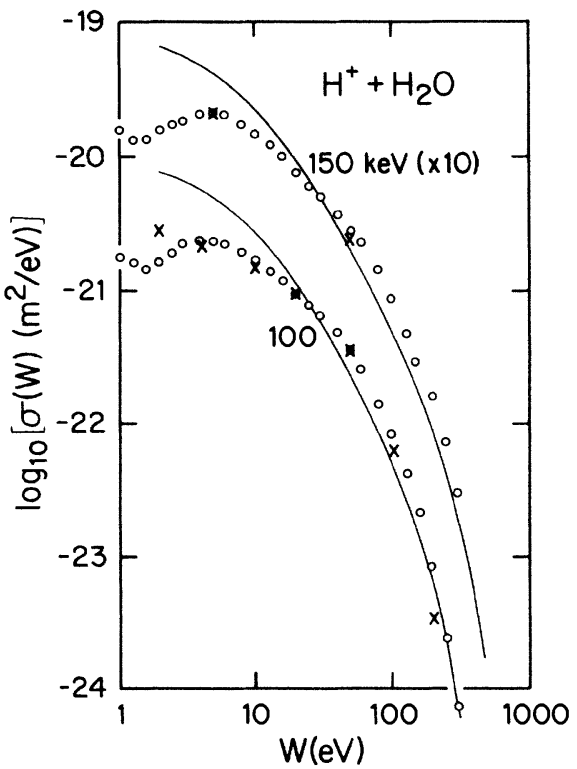

FIG. 5. Singly differential cross sections for ejection of electrons by 100 - and $150-\mathrm{keV}$ protons in water vapor as a function of electron energy. Circles, present data; $X$ 's, calculations by Senger et al. (Refs. 10 and 11); solid lines, BEA calculations. 


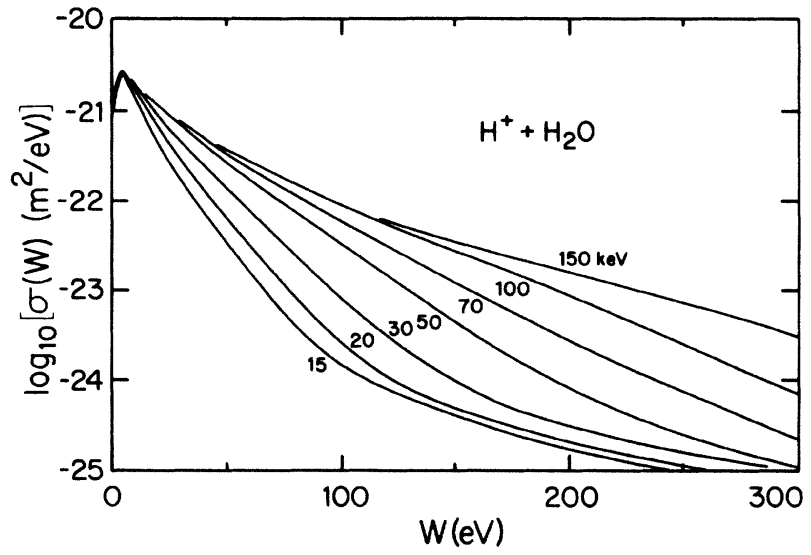

FIG. 6. Singly differential cross sections vs ejected electron energy for various proton energies.

without the target gas, there may have been a target pressure-dependent background which could not be eliminated in this way. This could, e.g., be due to uv photons generated in the collision region ${ }^{15}$ which managed to be reflected into the electron multiplier detector. This may account for the leveling off of the cross sections for low impact energy and high ejection energy. In earlier work ${ }^{4}$ using a different apparatus and different target gases, the low-energy cross sections decreased approximately exponentially with electron energy.

Total electron-ejection cross sections are shown in Fig. 7 compared to cross sections measured more directly which were reported earlier. ${ }^{16}$ While the present results are generally somewhat higher, the agreement is within the combined uncertainties of the two measurements.

\section{SCALING CROSS SECTIONS}

In an attempt to find relationships between electronejection cross sections for protons on different targets, we have tried two methods of scaling The first is based on the Born approximation and was given for SDCS by Rudd, Sautter, and Bailey. ${ }^{17}$ It may be written

$\sigma\left(W, E_{p}, B, N\right)=N(R / B)^{3} \sigma\left(W R / B, E_{p} R / B, R, 1\right)$,

where $N$ is the number of target electrons with binding energy $B, R$ is the Rydberg of energy, $W$ is the electronejection energy, and $E_{p}$ the proton-beam energy. This allows scaling from cross sections for atomic hydrogen to

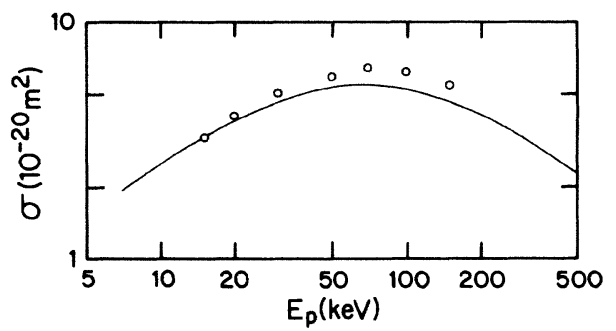

FIG. 7. Total electron-ejection cross sections vs proton energy. Circles, present data; solid line, data of Rudd et al. (Ref. 16).

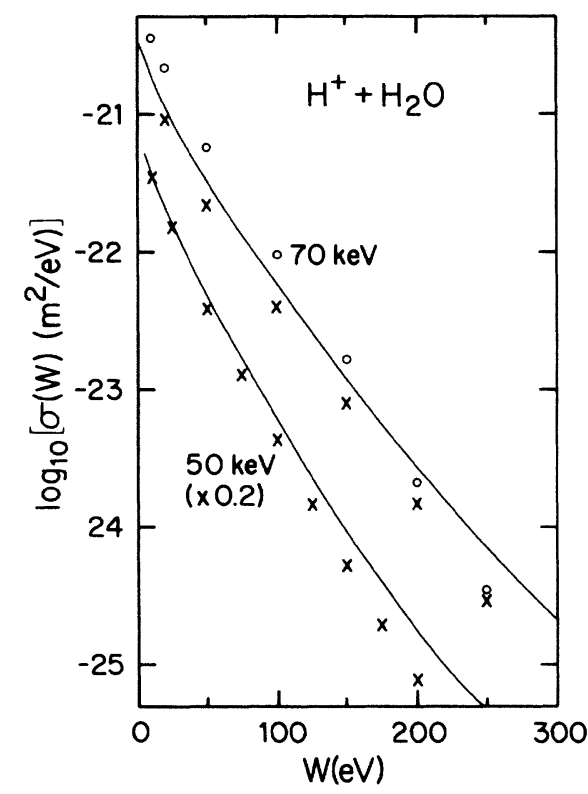

FIG. 8. Singly differential cross sections for 50- and 70-keV protons on water vapor. Solid lines, present experimental data; circles, results of scaling data on $\mathrm{H}_{2}$ (Ref. 4) by method described in text; $X$ 's, results of Bragg scaling using data on $\mathrm{H}_{2}$ (Ref. 4) and $\mathrm{O}_{2}$ (Ref. 19).

any other target provided the values of $N$ and $B$ for the various subshells of the target are known.

We have taken the data of Rudd ${ }^{4}$ for $\mathrm{H}_{2}$ and using $15.42 \mathrm{eV}$ as the ionization potential have scaled that to atomic hydrogen using Eq. (1) and then using the values of $N$ and $B$ for water vapor in the preceding paper ${ }^{18}$ scaled the hydrogen data to the various shells of water vapor. Approximations had to be made because data were not available at exactly the proton energies needed in the scaling; however, the error introduced should have only a small effect on the results. The results of this scaling are shown in Fig. 8.

Another method of scaling that has been applied to stopping powers is the Bragg rule for additivity. Applied to water it yields

$$
\sigma\left(\mathrm{H}_{2} \mathrm{O}\right)=\sigma\left(\mathrm{H}_{2}\right)+\frac{1}{2} \sigma\left(\mathrm{O}_{2}\right) .
$$

Calculations were made using the data of Rudd ${ }^{4}$ for $\mathrm{H}_{2}$ and the data of Crooks and Rudd ${ }^{19}$ for $\mathrm{O}_{2}$. These results are also shown in Fig. 8.

For this case, Bragg scaling yields reasonably good values at low ejection energies but gives values which are too low at higher energies. The other method is slightly better at high energies, but much worse for small values of $W$.

\section{ACKNOWLEDGMENTS}

This paper is based upon work supported by the National Science Foundation under Grant No. PHY-8025599. The authors wish to thank Bernard Senger for sending the results of his recent cross-section calculations. 
*Present address: Department of Physics, Shahid Bahonar (Kerman) University, Kerman, Iran.

${ }^{1}$ See, e.g., M. E. Rudd and J. H. Macek, Case Stud. At. Phys. 3, 47 (1972).

${ }^{2}$ See, e.g., Steven T. Manson, L. H. Toburen, D. H. Madison, and N. Stolterfoht, Phys. Rev. A 12, 60 (1975).

${ }^{3}$ T. L. Criswell, L. H. Toburen, and M. E. Rudd, Phys. Rev. A 16, 508 (1977).

${ }^{4}$ M. E. Rudd, Phys. Rev. A 20, 787 (1979).

${ }^{5}$ L. H. Toburen and W. E. Wilson, J. Chem. Phys. 66, 5202 (1977).

${ }^{6} \mathrm{M}$. Eugene Rudd and Theodore Jorgensen, Jr., Phys. Rev. 131, 666 (1963).

${ }^{7}$ R. K. Cacak and T. Jorgensen, Jr., Phys. Rev. A 2, 1322 (1970).

8J. Macek, Phys. Rev. A 1, 235 (1970).

${ }^{9}$ G. B. Crooks and M. E. Rudd, Phys. Rev. Lett. 25, 1599 (1970).
${ }^{10}$ Bernard Senger, Elisabeth Wittendorp-Reichmann, and Roger V. Rechenmann, Nucl. Instrum. Methods 194, 437 (1982).

${ }^{11}$ Bernard Senger and Roger V. Rechenmann, Nucl. Instrum. Methods B 2, 204 (1984).

${ }^{12}$ A. Salin, J. Phys. B 2, 631 (1969).

${ }^{13}$ L. Vriens, Proc. Phys. Soc. (London) 90, 935 (1967).

${ }^{14}$ M. E. Rudd, D. Gregoire, and J. B. Crooks, Phys. Rev. A 3, 1635 (1971).

${ }^{15}$ M. E. Rudd, J. Quant. Spectrosc. Radiat. Transfer 31, 387 (1984).

${ }^{16}$ M. E. Rudd, T. V. Goffe, R. D. DuBois, and L. H. Toburen, Phys. Rev. A 31, 492 (1985).

${ }^{17}$ M. E. Rudd, C. A. Sautter, and C. L. Bailey, Phys. Rev. 151, 20 (1966).

${ }^{18}$ M. A. Bolorizadeh and M. E. Rudd, preceding paper, Phys. Rev. A 33, 882 (1986).

19J. B. Crooks and M. E. Rudd, Phys. Rev. A 3, 1628 (1971). 